\title{
Ethical Issues in Conducting Research Involving Persons with Disability: A View from the Past and Some New Challenges
}

\author{
Diane Nelson Bryen ${ }^{1,2}$ \\ ${ }^{1}$ Centre for Augmentative and Alternative Communication, University of Pretoria, Faculty of Humanities, Pretoria, South Africa \\ ${ }^{2}$ College of Education, Temple University, Philadelphia, Pennsylvania, United States
}

\section{Email address:}

dianeb@temple.edu

\section{To cite this article:}

Diane Nelson Bryen. Ethical Issues in Conducting Research Involving Persons with Disability: A View from the Past and Some New Challenges. Humanities and Social Sciences. Special Issue: Ethical Sensitivity: A Multidisciplinary Approach.

Vol. 4, No. 2-1, 2016, pp. 53-59. doi: 10.11648/j.hss.s.2016040201.18

Received: January 25, 2016; Accepted: February 10, 2016; Published: May 13, 2016

\begin{abstract}
This paper traces the history of conducting research involving people with disabilities and raises serious questions about the ethics of conducting research. Ethical concerns include treatment of vulnerable populations, lack of informed consent, and benefit versus undue hardship when using people with disabilities as research participants. New technological advances, such as discussions on social media, present some new ethical concerns. Two case studies are presented that illustrate new ethical challenges. Guidelines are proposed that address the right to privacy, anonymity and confidentiality.
\end{abstract}

Keywords: Research, Ethics, People with Disabilities, Informed Consent, Beneficence, Non-maleficence

\section{Introduction}

Historically, people with disabilities and other vulnerable groups have been used as convenient research participants. Most of this research was in support of "medical progress" and raised serious questions about the ethics of conducting medical research. Many of these ethical concerns focused on the treatment of vulnerable populations, lack of informed consent, and benefits versus undue hardship when using people with disabilities as "subjects" of research. This paper traces some of the history of research conducted on people with disabilities as well as other vulnerable populations during the $19^{\text {th }}$ and $20^{\text {th }}$ centuries. However, given more recent advances in communication and information technologies in the early $21^{\text {st }}$ century, new research concerns are raised about ethical practices in conducting research involving people with disabilities.

\section{Some History Worth Knowing}

The quest for advancing scientific knowledge through human experimentation using vulnerable groups is traced back to ancient history, when Herophilus performed vivisections on prisoners [1]. In the $18^{\text {th }}$ century, Edward Jenner hypothesized that by injecting the less virulent cowpox vaccine in humans they would become immunized against the more virulent smallpox. On 14 May 1796 in England, Edward Jenner tested his hypothesis by inoculating James Phillips, a young boy of 8 years (the son of Jenner's gardener) with material from the cowpox blisters on the hand of Sarah Nelmes, a milkmaid who had caught cowpox from a cow called Blossom [2]. In addition to young James, Jenner tested his hypothesis on 23 subjects, most of whom were from lower social classes. It is unlikely that informed consent was obtained. By 1979, the World Health Organization declared that smallpox was an eradicated disease. Jenner's work is best known for discovering the vaccine against smallpox. However, today many researchers are likely to question the ethics of Jenner's methods based on the ethical concerns regarding testing vulnerable populations, failure to obtain informed consent, and weighing societal benefits in relationship to undue individual hardship.

In the late $19^{\text {th }}$ and early $20^{\text {th }}$ centuries, the sterilization of people with disabilities in the US began based on Darwin's theory of Evolution. Social Darwinism lead to the passage of the Eugenic Sterilization in the US Law of 1922 which 
formalized a program of sterilization of people with disabilities. According to Alex Wellerstein of Harvard University, the Eugenics Sterilization Law, written by Harry Laughton, resulted in the sterilization of more than 64,000 mentally ill and developmentally disabled patients by the time the law went into general disuse in the mid-1960s. Laughlin would later be awarded an honorary degree by the University of Heidelberg in 1936 for his work on behalf of the "science of racial cleansing." Based on the Eugenics Sterilization in the US Law, the Nazi German state sterilized thousands of people under their own sterilization laws [3].

Laughlin's Eugenics Sterilization Law was translated word-for-word and passed into German Law in 1933. By 1945, 330,000 Germans with disabilities were sterilized and by the end of World War II, many more were murdered in the German killing centers. According to the United States Holocaust Memorial Museum [4]:

"Within hours of their arrival at such centers, the victims [with disabilities] perished in especially designed gas chambers, disguised as shower facilities, utilizing pure carbon monoxide gas. Thereafter, T4 functionaries burned the bodies in crematoria attached to the gassing facilities. Other workers took the ashes of cremated victims from a common pile and placed them in urns to send to the relatives of the victims. The families or guardians of the victims received such an urn, along with a death certificate and other documentation, listing both a fictive cause and date of death."

"Because the program was secret, T-4 planners and functionaries took elaborate measures to conceal its deadly designs. Even though in every case, physicians and institutional administrators falsified official records to indicate that the victims died of natural causes, the "euthanasia" program quickly become an open secret. In view of widespread public knowledge of the measure and in the wake of private and public protests concerning the killings, especially from members of the German clergy, Hitler ordered a halt to the euthanasia program in late August 1941. According to T4's own internal calculations, the 'euthanasia' effort claimed the lives of 70,273 institutionalized mentally and physically disabled persons at the six gassing facilities between January 1940 and August 1941."

While there was a call for a halt to the T-4 action, a year later German medical professionals and healthcare works resumed the killings claiming the lives of 200,000 individuals with disabilities.

At the end of World War II, the Nuremberg Trials were held. According to the Nuremberg Trials Project [5]:

"The Medical Case, USA vs. Karl Brandt, et al. (also known as the Doctors' Trial), was prosecuted in 1946-47 against twenty-three doctors and administrators accused of organizing and participating in war crimes and crimes against humanity in the form of medical experiments and medical procedures inflicted on prisoners and civilians.

Karl Brandt, the lead defendant, was the senior medical official of the German government during World War
II...The specific crimes charged included more than twelve series of medical experiments concerning the effects of and treatments for high altitude conditions, freezing, malaria, poison gas, sulfanilamide, bone, muscle, and nerve regeneration, bone transplantation, saltwater consumption, epidemic jaundice, sterilization, typhus, poisons, and incendiary bombs.

These experiments were conducted on concentration camp inmates. Other crimes involved the killing of Jews for anatomical research, the killing of tubercular Poles, and the euthanasia of sick and disabled civilians in Germany and occupied territories. The defendants were charged with ordering, supervising, or coordinating criminal activities, as well as participating in them directly.

Karl Brandt and six other defendants were convicted, sentenced to death, and executed; nine defendants were convicted and sentenced to terms in prison; and seven defendants were acquitted [5]."

Those acquitted were medical personnel who had participated in deaths of Germans with disabilities but had not later participated in the concentration camp killings.

The most important result of the Doctors' Trial is the Nuremberg Code, which established a foundation for an ethical, legitimate research protocol involving human beings. Current research principles such as informed consent and absence of coercion, properly formulated scientific experimentation, and beneficence towards experiment participants are based on the Nuremberg Code. The full texts of these principles can be found in Trials of War Criminals before the Nuremberg Military Tribunals [6].

According to Annas [7], the Nuremberg Doctors' Trial marked the birth of American bioethics. Following the Nuremberg Code, the Helsinki Declaration on Human Research, and still later in 1979 the Belmont Report of the National Commission for the Protection of Human Subjects of Biomedical and Behavioral Research were built on the "bioethics born from the ashes of the Holocaust". In fact, according to Annas [7], the modern doctrine of informed consent was born at Nuremberg in 1947.

Despite the lessons learned from the Nuremberg Doctors' Trial in 1947, experimentation on people with disabilities and other "vulnerable" populations continued in the US ignoring the Nuremberg Code. Many of these experiments were conducted by well-educated physicians. How could this happen? One plausible explanation may be that when we view individuals or groups of individuals as less than human it is easy to perform inhuman acts without acknowledging that guilt [7]. Dehumanizing groups of people can partially explain why the Nazis expanded the American eugenics movement to the euthanasia of thousands of Germans with disabilities and later the murder of millions of Jews throughout Europe.

Germans with disabilities were considered "useless eaters." This is can be seen in the poster below used by the euthanasia propaganda published during Nazi Germany's Euthanasia Program officially spanning October 1939 until August 1941 but continued unofficially until the demise of 
the Nazi regime in 1945 and beyond [8, 9].

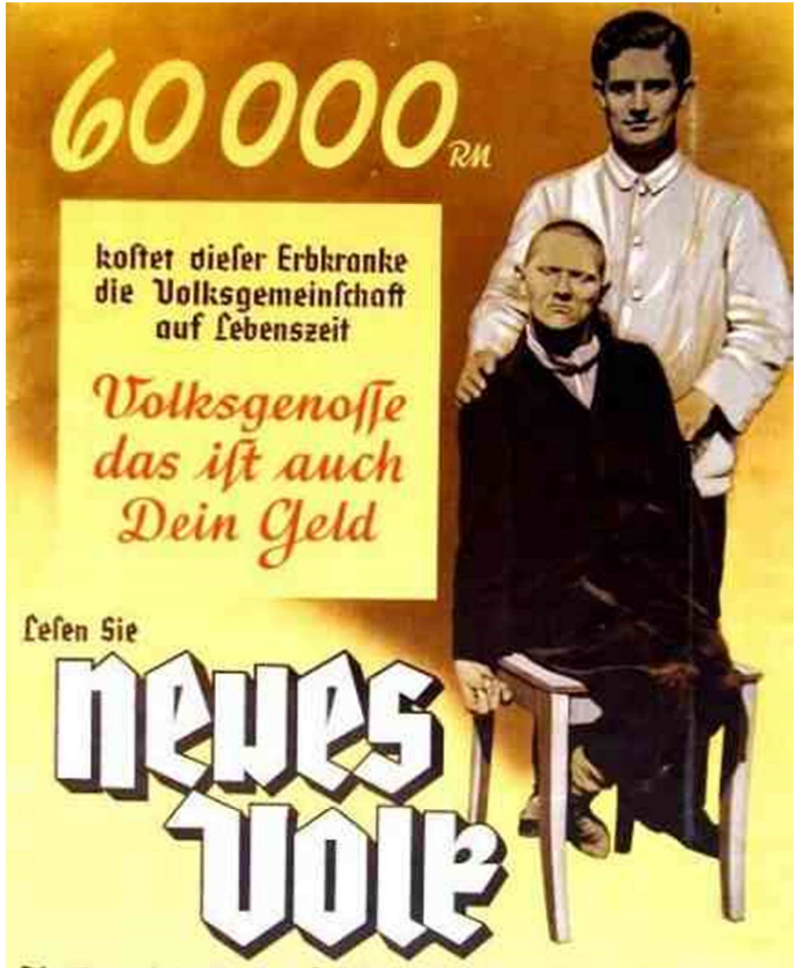

Die Monatshefte des Raffenpolitifchen Imtes der IISDaD

Figure 1. Propaganda Poster from the Nazi Party's policy of "acial hygiene."

This poster (circa 1938, with unknown author) reads: "60,000 Reichsmarks is what this person suffering from a hereditary disease costs the People's community during his lifetime. Comrade, that is your money too (Action T4, 2016)".

Propaganda posters, as the one shown above, were also used by the Third Reich to dehumanize Jews before sending them to the concentration camps. Dehumanizing Jews made it "easier" to carry out horrific experiments, such as:

- Infecting one twin with a "germ". When he/she died, the other twin was killed and their organs compared at autopsy;

- Sewing twins together to create a Siamese twin;

- Studying individuals with genetic defects and genetic traits so as to better "purify the Aryan super race;"

- Performing cross transfusions to "make boys into girls and girls into boys;" and

- Freezing experiments [10].

In the US, human radiation experiments were preformed on school children with intellectual disabilities. No informed consent was provided by the parents and the children were "induced" to participate even if they refused.

In 1952, during the Polio scare in the US, Jonas Salk injected 43 children with his killed Poliovirus vaccine at the D.T. Watson Home for Crippled Children. Salk also injected children at the Polk State School for the "retarded and feeble minded" during the same year [11].

Studies of hepatitis were conducted on children with intellectual disability who were residing in a large, segregated institution in New York, called the Willow brook State School, which was closed in 1987. Inadequate consent was provided when infecting children living there. This highly controversial medical study carried out between 1963 and 1966 by medical researchers involved healthy children who were intentionally inoculated, orally and by injection, with the virus that causes the disease, then monitored to gauge the effects of gamma globulin in combating it [12].

\section{Ethics in Disability Research}

The Nuremberg Code in 1947, the Helsinki Declaration in 1964, and the Belmont Report in 1985 form that basis of all US research regulations implemented by the Department of Health and Human Services (DHHS), the Federal Drug Administration, and the Department of Education [13]. Special protections under DHHS regulations focus on three "vulnerable" populations: (a) fetuses, pregnant women, and human in vitro fertilization; (b) prisoners; and (c) children. Note that individuals with disabilities are NOT included as needing special protections in research based on being a "vulnerable" population. As such, this is one of the major problems with research ethics in the US. It may account for the more recent violations of the Nuremberg Code (need for informed consent, knowingly harming research participants by deliberately infecting them). Furthermore, unethical research using individuals with disabilities continues even with Institutional Review Board (IRB) oversight [13].

When people with disabilities are stigmatized and isolated, there is a great risk that they will be viewed as less that human [11]. As such, the regulations that guide research practices may be overlooked by researchers. In response to this problem, Dr. Anne Good of the National Disability Authority has published an important paper entitled "Ethics in Disability Research [14]." Based on the United Nations standards on the Equalization of Opportunities for Persons with Disabilities (1993) and the 1996 report of the Irish Commission on the Status of People with Disabilities, Good suggests the following ethical guidelines focused specifically on research involving persons with disabilities:

- Respect for the human rights, dignity, equality and diversity of all involved in the research process

- Advancement of social justice for people with disabilities within the wider community

- Promotion of the well-being of all participating, involved in or affected by the research process

- Avoidance of harm to those involved in the research process or to the wider community

- Facilitation of the participation of people with disabilities in research and research dissemination, including those for whom obstacles might make such participation difficult without additional support

- Maintenance of the highest professional, legal and ethical standards and competencies

- Comprehension and fulfillment of relevant legal responsibilities [14]. 
- These seven guidelines are then translated into five research practices that should ensure ethical research:

- Well-being (beneficence) and avoidance of harm (nonmaleficence)

- Collaboration with people with disabilities in conducting research

- Consent: Informed and voluntary

- Respect for participants: Dignity, anonymity, and privacy, and

- Equality and diversity among people included in the research design and planning

Farrelly [15] provides additional requirements regarding ethical research practices involving people with disabilities. Two of these requirements are summarized below.

Recruitment. In order to ensure that potential research participants understand that they do not have to participate in a study, clearly state using Easy English ${ }^{1}$ that participation is voluntary. Tell them that they do not have to give a reason for declining to participate, and that declining will not interfere with their treatment or services or harm them in any possible way. Finally, state that they can withdraw from the research at any time without any negative consequences.

Consent. In order to ensure voluntary and informed consent, the researcher must provide a simple description of the purpose and the procedures of study. Furthermore, the researcher should explain simply and clearly how the study will benefit the individual, the length and location of the study procedures, and any adverse effects which might occur. Additional information must include how the research results will be disseminated, how and when the person will get a summary of the results in an understandable format, how confidentiality will be ensured, and assurance that the person can withdraw at any time. Finally, the individual giving consent and the researcher should both sign the consent form and keep a copy. An example of a consent form using Easy English with graphic symbols (Picture Communication Symbols) are shown in the Appendix.

\section{New Challenges}

The widespread development and use of generic and specialized electronic technologies has opened enormous opportunities for children and adults with disabilities. Communication and information technologies enable individuals with disabilities to communicate across time and space and to obtain information at any time and from any place. Online discussion boards, consumer health information, and online support groups show great promise [17]. Speech generating devices enable people with disabilities who have little or no functional speech to communicate in a variety of contexts for a variety of

1 Easy English is a more accessible style of English for people who have difficulty reading and understanding written information. It uses clear and simple language, one idea per sentence, short sentences, direct language (readers are addressed as "you", pictures or photographs to add meaning to the text, and minimum punctuation [16] purposes and with a variety of audiences.

With these new technological advances come new challenges to research. Flicker et al. [17] note three research situations that raise significant ethical predicaments: “(a) enrolling research participants, (b) protecting participants from risk or harm, and (c) linking public and private data." These ethical predicaments are of even greater concern when conducting research on vulnerable populations including those with disabilities. Two case examples in which the author has personal familiarity highlight these ethical dilemmas. Procedures to ensure the centrality of the ethical principle of autonomy articulated in the Declaration of Helsinki are also suggested.

\section{Case Studies}

\subsection{Case Study 1}

Recruitment of research participants with low incidence disabilities can be problematic and often costly in time. When there is a "captive audience", such as school, institution or an electronic mailing list (listserv), recruitment is much easier. However, this recruitment opportunity raises potential ethical violations. One such situation is that of recruiting potential participants from an online support group or using archival material from that listserv. Online support groups, such as those who have survived sexual abuse or recovering addicts, assume privacy and confidentiality - a distinction being made by what "data" is private and what is public [18].

One such online support group involves people with disabilities. It is not moderated and welcomes parents and professionals. From time to time, professionals want to recruit research participants from this online community or use the information from more than 2 decades that is available to subscribers in the listserv archives. The vast amount of information that is available in these archives has made this listserv a prime target for researchers wishing to study this particular group of individuals. Recruiting research participants from this convenient pool of subscribers is enticing for researchers since they can collect data from a widely dispersed population at relatively low cost and in less time than traditional recruitment methods would require. Furthermore, according to Frankel and Siang [19], "the ability of both researchers and their research participants to assume anonymous or pseudonymous identities online, the complexities of obtaining information consent, the often exaggerated expectations, if not the illusion, of privacy in cyberspace, and the blurred distinction between public and primate domains fuel questions about the interpretation and applicability of current policies governing the conduct of social and behavior research involving human subjects $[19, \mathrm{p}$. 1-2]." Frankel and Siang [19] question how the basic ethics principles of autonomy and informed consent are protected. How are the risks and benefits of the research disclosed to the subject? How is privacy and confidentiality protected? How is the principle of beneficence involving maximizing 
the benefits for the subject while the principle of nonmaleficence (minimizing the possible risks resulting from the research) ensured?

In an attempt to address some of these ethical predicaments, the human listserv administrator in collaboration with several subscribers with disabilities, developed an explicit policy about research involving this specific online community. The policy states:

Research is not permitted on the Listserv ${ }^{2}$ for several reasons. First, there have been many requests to conduct research on the Listserv or to use subscribers of Listserv as a convenient subject pool. Second, this Listserv is partially funded by a Grant $^{3}$ as a platform for information dissemination and not for research purposes. As such, use of the Listserv for research purposes would firstly jeopardize the trust and confidentiality of the members of the Listserv and secondly violate the basis for its funding.

Here are what members of the Listserv have suggested as reasonable and respectful procedures for research connected with this Listserv. Researchers can provide a short description of the purpose of their research project (for informational purposes only) on the Listserv with a link to a website that provides greater detail. This way members of the Listserv will learn about your research through the Listserv and then decide if they want to go to that website to learn more about it. Information provided on the researcher's website must include the following:

1. Details of the study (e.g., purpose, funding, sample, instrumentation) in Easy English;

2. IRB/ human protections approval/confidentiality with the IRB approval Number;

3. Description of what the researcher want "subjects" to do, time involved, risks, procedures to ensure confidentiality and compensation in Easy English;

4. Actual study protocol, accessible formats, etc.;

5. How results will be used and disseminated to the participants; and

6. Consent letter in Easy English to be signed, electronically, if needed.

7. No mention of individual names nor the name of the Listserv is to be used in any publication of the research.

This research policy was developed to respect the people who make up these important online communities and their right to privacy and confidentiality. It was also developed to support the autonomy and diversity of the members with disability of this online community.

\subsection{Case Study 2}

Many of the new communication technologies generally referred to as assistive communication devices or speech generating devices (SGD) are used to augment and support

2 The name of the listserv is not being provided in order to protect the privacy and anonymity of the subscribers. Instead, it is simply referred to as "Listserv."

3 The particular funding source is purposely unnamed and is simply referred to as the "Grant." This is done once again to protect the privacy of the members of this online community. spoken language of people with little or no functional speech. Most of these computer-based devices have large buffers that can store recorded messages generated by the person using. According to Hill and Romich [20], language activity monitoring (LAM) is the systematic data collection of the actual language activity of an individual with limited or no functional speech who relies on augmentative and alternative communication (AAC). The authors state that the use of the LAM is to provide support for evidencebased clinical intervention and research outcomes [21]. The LAM is a standard feature built into some SGDs or as an add-on package for others. It can record the time and content of language events produced by the person using the SGD. However, the language events of the conversational partners or the context of the conversation is not recorded.

Several ethical questions arise here. For example, how are the principles of autonomy and informed consent protected? How are the risks and benefits of the use of LAM data disclosed to the subject? How is the principle of beneficence involving maximizing the benefits for the subject while minimizing the possible risks resulting from the research? How is privacy and confidentiality ensured?

With these ethical questions in mind, it is proposed that the default on LAM software or the add-on software be "disengaged" on the individual's SGD. If the professional supporting the individual has reason to monitor the language activity of an individual for whatever clinical, developmental, training, or research purposes, the five research practices described earlier in this paper by Good [14] should be implemented and approved before the LAM is "engaged".

As in the previous case study, this guideline is proposed in order to respect the individuals who use these communication devices as their spoken language and their right to privacy, anonymity and confidentiality.

\section{Conclusion}

This paper provides a brief history of the development of legal and ethical practices for conducting research on vulnerable populations including children and adults with disabilities. Out of the ashes of the Holocaust, American and international guidelines, codes, and regulations have been developed to protect the autonomy, beneficence, and privacy of people with disabilities without denying them the opportunity to participate in research that would benefit them. New technologies that improve the independence, participation, and contributions of people with disabilities also present new threats to ethical research practices. It is now up to all of us - researchers, advocates and allies, providers of services and supports, and people with disabilities themselves - to apply these principles of ethical conduct in all of our research activities. Research practices focused on people with disabilities should reflect the cry "nothing about me without me." 


\section{Appendix}

Sample Consent Form Using Easy English and Pictures ${ }^{4}$

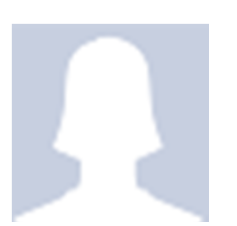

\section{Information for Sally Joe}

My name is Diane and I would like you to be part of my project.

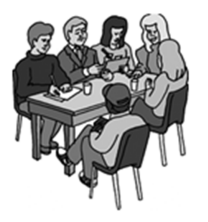

My project is to find out more about making friends and communication

I want to spend time with you.
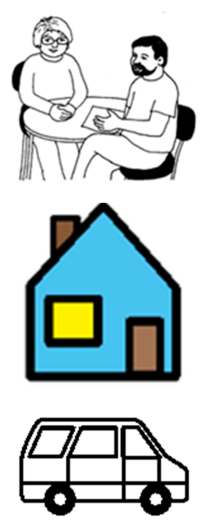

I want to go out with you

I want to take your photo

I will only use your picture if you say yes

What happens with your information?

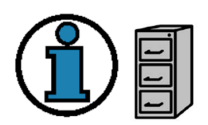

I will keep this in a safe place for 7 years. I will not use your name or other information that shows who you are.

You can stop anytime and say NO.

When I have finished. I will say goodbye

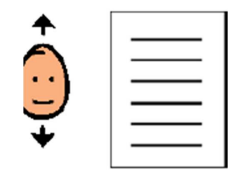

I say yes to the information in this

form.

$--/-1-$ The date today. Write the day, month and year.

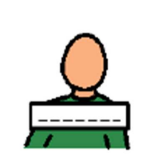

My name

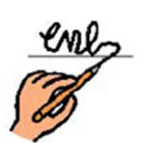

My signature

OR

You can ask a person to sign for you.

The person must be legally allowed to sign for you. This person is called your representative. Turn the page.

Representative name

Representative's signature

Also, a witness must sign this form.

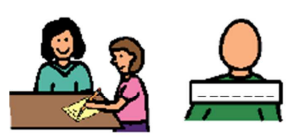

Witness name

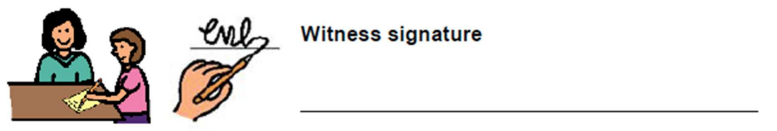

4 This sample consent form is based on the work of Hilary Johnson of the SCOPE Centre in Melbourne, Australia. Used with permission. 


\section{References}

[1] Von Stodden, H. (1989). Herophilus: The art of medicine in early Alexandria. Cambridge University Press: Cambridge.

[2] Jenner, E. (1969), Vaccination against smallpox. Great Mind Series, Prometheus Books.

[3] Wellerstein, A. (2011) Harry Laughlin's "Model Eugenical Sterilization Law". Retrieved from http://www.people.fas.harvard.edu/ wellerst/laughlin/ on May $18,2011$.

[4] United States Holocaust Memorial Museum. "Euthanasia Program." Holocaust Encyclopedia. http://www.ushmm.org/wlc/en/?ModuleId=10005200. Accessed on January 24, 2016.

[5] Nuremberg Trials Project (2003). Introduction to NMT Case 1, U. S. A. v. Karl Brandt et al. Harvard Law School Library retrieved from

http://nuremberg.law.harvard.edu/php/docs_swi.php?DI=1\&te $\mathrm{xt}=$ medical on January 24, 2016.

[6] Trials of War Criminals before the Nuremberg Military Tribunals under Control Council Law No. 10, Vol. 2, Washington, D. C.: U. S. Government Printing Office, 1949, pages $181-182$.

[7] Annas, G. T (2009). The legacy of the Nuremburg doctors' trial to American bioethics and human rights. MINN. J. L. SCI \& Tech. 10 (1), 19-40.

[8] Browning, C. The Origins of the Final Solution: The Evolution of Nazi Jewish Policy, September 1939 - March 1942, p. 193 found in Wikipedia http://en.wikipedia.org/wiki/Action_T4\#cite_note-1 on January 24, 2016.

[9] Lifton, R. J. THE NAZI DOCTORS: Medical Killing and the Psychology of Genocide, p. 95-96, found in Wikipedia. http://en.wikipedia.org/wiki/Action_T4\#cite_note-1 on January 24, 2016.

[10] Annas, G., \& Grodin, M. (1992) The Nazi Doctors and the Nuremberg Code: Human Rights in Human Experimentation. New York: Oxford University Press. pp. 53-59. ISBN 978-019-510106-5.

[11] Keefer, A. (2011). Uninformed consent: Medical experimentation on vulnerable populations. In D. N. Bryen \&
A. R. Keefer (eds). A guide to embedding disability students into the humanities. Temple University. Unpublished manuscript.

[12] Hevesi, D. (2010). "Robert W. McCollum, Dean of Dartmouth Medical School, Dies at 85", The New York Times, September 25, 2010. Accessed May 25, 2011.

[13] Research (2011). Problems with human subject experimentation: Relationship to codes of research and ethics. Retrieved from research.utmb.edu/IRB/files/Ethics.pps on May 26, 2011.

[14] Good, A. (2005). Ethics in disability research. NDA Ethical Guidelines for Disability Research. Retrieved from http://www.nda.ie/cntmgmtnew.nsf/0/D6EFA30A02A47B148 $02570660054 \mathrm{EC} 16$ ? OpenDocument on May 26, 2011.

[15] Farrelly, M (2004). Ethics in research with people with disabilities. Annual PSI Conference, November, 2004. Retrieved from www.psihq.ie/DOCUMENTS/ERLD.PDF on May 27, 2011.

[16] Victoria Department of Justice. (2011) Easy English fact sheets. Retrieved from http://wwwlconsumer.vic.gov.au/CA on May 30, 2011.

[17] Flicker, S., Haans, D., \& Skinner, H. (2004). Ethical dilemmas in research on internet communities. Qualitative Heath Research, 14(1), 124-134.

[18] King, S. A. (1996). Researching online communities. Proposed ethical guidelines for the reporting of results. The Information Society - An International Journal. Vol 12, 2, 119-128.

[19] Frankel, M. S. \& Siang, S. (1999). Ethical and legal aspects of human subjects research on the internet. American Association for the Advancement of Science. Retrieved from http://www.aaas.org/spp/dspp/sfrl/projects/intres/main.htm on May 27, 2011.

[20] Hill, K. \& Romich, B. (2001). A language activity monitor for supporting AAC evidence-based clinical practice, Assistive Technology, 13 (1), 12 -22.

[21] Hill, K. \& Romich, B. (1999). AAC language activity monitoring and analysis for clinical intervention and research outcomes. Paper presented at the 1999 CSUN conference. Retrieved from http://www.dinf.ne.jp/doc/english/Us_Eu/conf/csun_99/sessio n0063.html on January 24, 2016. 